However, factors that influence vaginal bacterial community composition and dynamics are not well understood. There have been conflicting reports of altered vaginal microbiota and infection susceptibility with contraception use. The objective of this study was to determine if contraception use altered the vaginal microbiota.

Methods Vaginal swab samples were obtained from over 400 women during their first year of using hormonal contraception (levonorgestrel intrauterine system (LNG-IUS), depot medroxyprogesterone acetate (DMPA), combined oral contraceptive, contraceptive patch and etonogestrel implant) or a non-hormonal copper intrauterine device. Samples were obtained at baseline, 6 months and 12 months as part of the Contraceptive CHOICE study. The V4 region of the bacterial 16S rRNA-encoding gene was amplified from the vaginal swab DNA and sequenced with an Illumina MiSeq. The $16 \mathrm{~S}$ rRNA gene sequences were processed and analysed using the software package mothur. After clustering the sequences into operational taxonomic units (OTUs) based on sequence similarity we calculated several ecological metrics including $\theta_{\mathrm{YC}}$ distances (a metric that takes relative abundances of both shared and non-shared OTUs into account) between communities.

Results The vaginal microbiota in this study clustered into 3 major vaginal bacterial community types: one dominated by Lactobacillus iners, one dominated by Lactobacillus crispatus and one more diverse community type. Initial analysis indicates differences between the microbiota at baseline and after LNG-IUS use.

Additionally, specific OTUs were enriched with the use of certain contraceptive methods. For example, higher levels of 2 Prevotella OTUs were associated with DMPA use.

Conclusion Alterations of the vaginal microbiota are associated with the use of certain contraceptives. Further studies and analysis will be needed to verify these findings and determine the implications for infection susceptibility.

Disclosure of interest statement We did not receive any commercial contributions for this study.

\section{P06.08 THE ASSOCIATION BETWEEN FREE GLYCOGEN IN THE VAGINAL FLUID AND COLONISATION BY LACTOBACILLI}

${ }^{1}$ MA Beamer*, 'LA Meyn, ${ }^{1}$ LK Rabe, ${ }^{1}$ S Hendrickson, ${ }^{1} \mathrm{HA}$ Avolia, ${ }^{1} \mathrm{MN}$ Austin, ${ }^{1,2} \mathrm{~K}$ Bunge, 1,2 BJ Moncla, ${ }^{1,2}$ SL Hillier. 'Magee-Womens Research Institute; ${ }^{2}$ University of Pittsburgh Department of Obstetrics, Gynecology and Reproductive Sciences, Pittsburgh, PA, USA

10.1136/sextrans-2015-052270.309

Introduction Glycogen is both an energy source and metabolic product of lactobacilli. Our objective was to assess the association between free glycogen in the cervicovaginal lavage (CVL) and vaginal microbiota as assessed by Nugent score, quantitative polymerase chain reaction (qPCR) for Lactobacillus crispatus and L.iners, and quantitative culture detection of lactobacilli.

Methods Healthy women $(\mathrm{n}=55)$ aged $18-45$ without clinical bacterial vaginosis, gonorrhoea, chlamydia or trichomoniasis were enrolled. A $10 \mathrm{~mL} \mathrm{CVL} \mathrm{sample} \mathrm{was} \mathrm{collected} \mathrm{and} \mathrm{tested}$ for glycogen using a fluorometric assay (BioVision) and protein was assessed using the Lowry assay. A vaginal smear was interpreted using the Nugent criteria. Separate vaginal swabs were used for vaginal culture and qPCR. Differences in median levels of glycogen were evaluated using the Kruskal-Wallis test.

Results Glycogen concentrations (ng/ug protein) were significantly higher in women having a Nugent score of 0-3, compared to those having scores of 4-6, or 7-10 (457 vs 398 vs $128, \mathrm{P}=0.049$ ). Glycogen content was higher among women colonised by $L$ crispatus vs other lactobacilli (L. jensenii, L. gasseri, L. iners) vs no lactobacilli (426 vs 280 vs $36, \mathrm{p}=0.013$ ) based on culture. Similarly, the 38 women having L. crispatus dominant $\left(>10^{5}\right)$ flora by qPCR had higher glycogen levels vs the 15 women who had dominant $L$ iners (413 vs $201, \mathrm{P}=$ 0.036).

Conclusion Increased levels of free glycogen in the CVL are associated with flora dominated by L. crispatus. It is unknown whether glycogen enhances $L$. crispatus colonisation, or whether L. crispatus synthesises glycogen, increasing the glycogen content.

Disclosure of interest statement Nothing to declare.

\section{P06.09 LACTOBACILLUS CRISPATUS INHIBITS GROWTH OF GARDNERELLA VAGINALIS AND NEISSERIA GONORRHOEAE ON A PORCINE VAGINAL MUCOSA MODEL}

${ }^{1}$ LM Breshears*, ${ }^{2}$ VL Edwards, ${ }^{2} \mathrm{~J}$ Ravel, ${ }^{1} \mathrm{ML}$ Peterson. ${ }^{1}$ University of Minnesota, College of Pharmacy, Department of Experimental and Clinical Pharmacology, Minneapolis, Minnesota, USA; ${ }^{2}$ University of Maryland School of Medicine, Institute for Genome Sciences, Baltimore, Maryland, USA

\subsection{6/sextrans-2015-052270.310}

Introduction The vaginal microbiota affects susceptibility to bacterial vaginosis (BV) and sexually transmitted infections (STIs). $\mathrm{BV}$ is characterised by depletion of Lactobacillus spp., an overgrowth of anaerobes (usually dominated by Gardnerella vaginalis) and a $\mathrm{pH}>4.5$. BV is associated with an increased risk of acquiring STIs such as chlamydia and gonorrhoea. An ex vivo porcine vaginal mucosal model (PVM) was developed to explore the mechanistic role of Lactobacillus in affecting vaginal colonisation by G. vaginalis and Neisseria gonorrhoeae.

Methods Explants $(5 \mathrm{~mm})$ of freshly collected PVM were placed in transwells over various media, including Lactobacillus culture supernatant, inoculated with bacteria and incubated under aerobic or anaerobic conditions. Colonised explants were processed for CFU enumeration and presence of biofilm (via confocal microscopy) at indicated times. Lactic acid produced by a clinical isolate of L. crispatus growing on PVM was also quantified.

Results All isolates tested could colonise and grow on PVM. G. vaginalis and $N$. gonorrhoeae form biofilms on PVM. L. crispatus produces lactic acid on PVM and inhibits the growth of $N$. gonorrhoeae and G. vaginalis in a $\mathrm{pH}$-dependent manner. Finally, L. crispatus produces a secreted factor that kills $N$. gonorrhoeae on PVM at an otherwise permissible $\mathrm{pH}$.

Conclusion These data demonstrate that PVM is a useful model for studying the interactions of commensal vaginal microbes with pathogens on the vaginal mucosa. The data confirm a role for lactic acid in inhibiting growth of G. vaginalis and N. gonorrhoeae. The discovery of an L. crispatus secreted factor that kills $N$. gonorrhoeae is intriguing and future work will identify this compound and explore its mechanism of action.

Disclosure of interest statement Funding provided by the Office of the Vice President for Research, University of Minnesota and NIH grant U19AI084044. No pharmaceutical grants were received in the development of this study. 